\title{
AN OPENBIM BASED DATA MAPPING METHODOLOGY TOWARDS STRUCTURAL ANALYSIS USING FEM
}

\author{
Fangzheng $\operatorname{Lin}^{1}$ and Raimar J. Scherer ${ }^{2}$
}

\begin{abstract}
In terms of building structural analysis, a large number of researchers throw the spotlight on data swapping from Industry Foundation Classes (IFC) models to input files from various kinds of Finite-Element-Analysis software. These attempts succeeded in accelerating pre-processing before simulation. However, the associated methodologies do not break this restriction of data-to-data mapping. Up to now, an openBIM based mapping methodology describing software-independent data mapping form an IFC model to a Finite-Element system emerges yet hardly in publications. This paper explores the theoretical possibility to automate data mapping from models standardized by IFC directly to the equilibrium equations for structural analysis with the Finite Element Method (FEM). For this purpose, a semantic model towards structural analysis using beam elements or plane elements is established to represent the topology of corresponding objects based on the data analysis result. Subsequently, the conception of data mapping is elaborated through a conceptual case study.
\end{abstract}

Keywords: OpenBIM, Industry Foundation Classes, Finite Element Method, Structural analysis.

\section{INTRODUCTION}

Building Information Modelling (BIM) is put forward firstly in the middle of the 20th century (Quirk 2012). This concept arises along with the ongoing digitization of the entire Architecture, Engineering and Construction (AEC) industry. Building elements and their topological relations can be semantically represented in a BIM model. For a better sharing possibility of BIM models, the initiative of openBIM was proposed by several leading software vendors (GRAPHISOFT 2020). The Industry Foundation Classes (IFC) schema developed and maintained by buildingSMART is one of the standards in the frame of openBIM, and is already widely supported by the mainstream BIM software applications (Revit, Allplan, Bently, etc.) in the field of Computer-Aided Design/Engineering. IFC standard is designed for the objective of interoperability, so that the data exchange and sharing could break through the barriers between software applications or platforms.

BIM models generated based on IFC schema are named as IFC models. This interoperability-oriented IFC models demonstrate a broad spectrum of application. Since early years of this century, the integration technology of IFC/BIM and Geo-graphic Information System has always been under development. The corresponding studied

1 Research Associate and PhD Student, Institute of Construction Informatics, Technische Universität Dresden, Dresden, Germany, fangzheng.lin@tu-dresden.de

2 Senior Professor, Institute of Construction Informatics, Technische Universität Dresden, Dresden, Germany, raimar.scherer@tu-dresden.de 
objects could be a single building (Thiis and Hjelseth 2008), indoor modelling (Choi et al. 2008), urban management (Yamamura et al. 2017), etc. In the domain of Facility Management, IFC also becomes a popular topic for more convenient data acquisition and more effective object visualization (Fu et al. 2006). Internet of Things is dedicated in information communication. In terms of its implementation, Motamedi et al. demonstrated the applicability of the proposed method using IFC and Radio Frequency Identification through a real-world case study (Motamedi et al. 2016).

Besides the proceeding research work, BIM oriented simulation known as BIM2SIM is of concern, as well, for the researchers trying to broaden the application domains of IFC. Andriamamonjy et al. proposed a work flow to filter the information from related building elements out, and to convert them to structure data files for the engine of Building Energy Performance Simulation (Andriamamonjy et al. 2018). For certain energy simulation software, an interface could be programmed to automate the data exchange from an IFC model to an input file (Ahn et al. 2014). Similarly, Dimyadi succeeded in transforming an IFC model to an input file for fire and smoke simulation (Dimyadi et al. 2008).

As a matter of fact, the core of so called simulation is numerical computation using Finite-Element-Method (FEM), which can serve not only for thermal calculation (e.g. energy simulation) and fluid dynamics (e.g. fire simulation), but also for structural analysis. Romberg et al. attempted transforming slabs and columns from an IFC model to a FEM model mesh by solid elements (Romberg et al. 2004). Hu et al. completed a data ex-change mechanism from IFC models to several input models from FEA software and among these input models (Hu et al. 2016). The concept Building information modelling ought to specifically refer to buildings, however, the idea of information modelling has been extended to infrastructures as the representative of other structure categories. Stascheit et al. studied the data requirements in simulating a conventional tunnel structure in an open source simulation framework and competed the data mapping from an IFC tunnel model to the input model in pre-processing of FEA software (Stascheit et al. 2013). The alike data exchange work flow was also preliminary automated by Hamdan for a bridge project (Hamdan 2018).

So far, the majority of articles corresponding to BIM2SIM threw light on information transformation from corresponding building elements in IFC building models to the input files of simulation software. Each kind of simulation software has its own modelling philosophy and a specific compiler to make its simulation engine understanding the input files. Due to these reasons, related methodologies can only be implemented separately with certain software. By means of that, the simulation engine developed obeying the principle of FEM is barely touched during the procedure of model transformation. Therefore, it makes sense to bridge the gap directly between IFC models and the bottom algorithm of FEM (Figure 1).

The above mentioned issue of hereby the focus of this paper. The data architecture of IFC models for structural analysis is requested to be analysed. An equilibrium equation system using FEM needs to be established and the data requirements equation system should be investigated. Through a conceived semantic model and a conceptual case study, the FEA-software-independent data exchange process is discussed and implemented. 


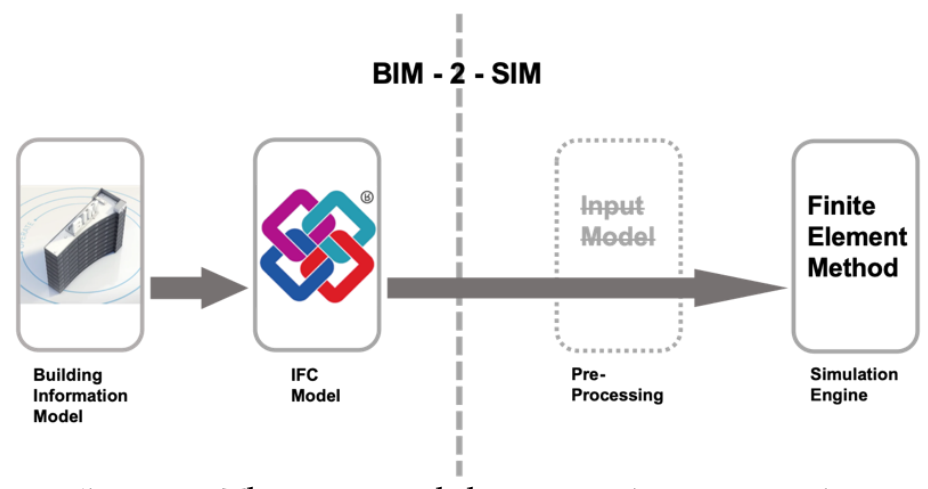

Figure 1: The proposed data mapping conception

\section{ANALYSIS OF IFC STANDARD}

For the purpose of data swapping in this paper, the domain of structural analysis in IFC standard and the related IFC objects will be studied. The essential entities for structural analysis was defined by the completed IAI project "ST-4" (Weise et al. 2003), emerged intuitively in IFC $2 \times 2$, and has been inherited to the current IFC version (IFC 4). Figure 2 demonstrates typical IFC objects/entities for structural analysis. While IFC curve members represent columns, walls and slabs are defined as surface elements. It is not demonstrated in Figure 2, but reasonable to infer that curve members and surface members can also respectively instanced though beams and plates. A complete structure is usually assembled by several kinds of structural elements, thus the continuity between these elements are implemented by connection definition including point connection and curve connection. This connection definition also belongs to element coupling from the point of view of FEM. In this section, the data structure of related building elements and element connection are requested to be investigated.

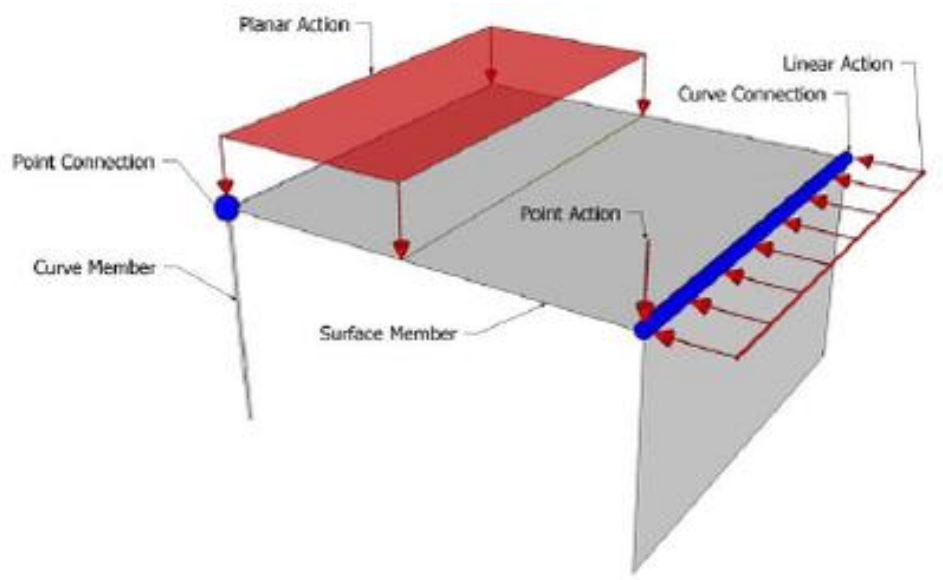

Figure 2: Visualization of a typical structural analysis model from IFC

(buildingSMART 2020a)

\subsection{Building Objects}

\subsubsection{Beam and Column}

Beams and columns are usually meshed by beam elements in structural analysis. Considering the features of beam elements, the essential information that IFC models are 
capable to deliver to the equilibrium equation of structural analysis encompasses Young's modulus $E$, length of beam element $l$ and moment of inertia $I$. $E$ is a constant designated in the model attribute of material; $l$ indicates the offset of cross sections' sweep or extrusion that can be simply obtained from coordinate values in IFC models; $I$ as one of cross section geometric coefficients must be calculated based on the given cross section profile. In view of modelling building beams or columns according to IFC standard, the proceeding required information originate from the two objects namely axis and profile. Therefore, the definitions corresponding to both objects in IFC standard are analysed.

As the domain of structural analysis in IFC standard states, beams and columns belong to the entity IfcStructuralCurveMember. Figure 3 and Figure 4 illustrate the data architecture of axis and profile definitions. The entity IfcEdge, composed of a starting IfcVertexPoint and an end IfcVertexPoint, serves for axis modelling of the related IfcStructralCurveMember. IfcVertexPoints are quantified through 3D coordinates contained by IfcCartesionPoints. IfcDirection indicates vectors from the starting point to the end point. IfcMaterial is the entity, where material names and parameters can be designated through property sets based on IFC standard. IfcProfileDef is a super-type of all definitions of standard and arbitrary profiles, the entity is used to define a standard set of commonly used section profiles by parameters or explicit curve geometry (buildingSMART 2020b). The majority of typical profile categories for steel and reinforced concrete structure are included among the sub-types of IfcProfileDef, e.g. IfcRectangle-HollowProfileDef, IfcRectangleProfileDef, IfcIShapeProfileDef, IfcTShapeProfileDef, etc.

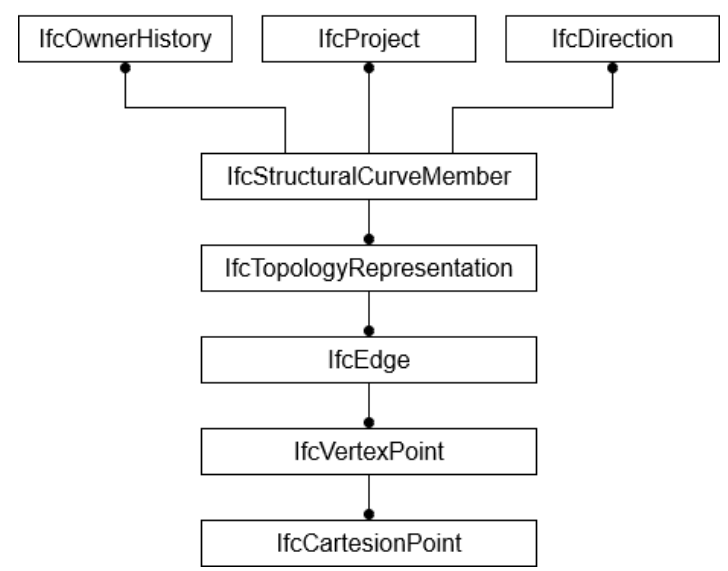

Figure 3: Referenced entities for axis definition in IfcStructuralCurveMember 


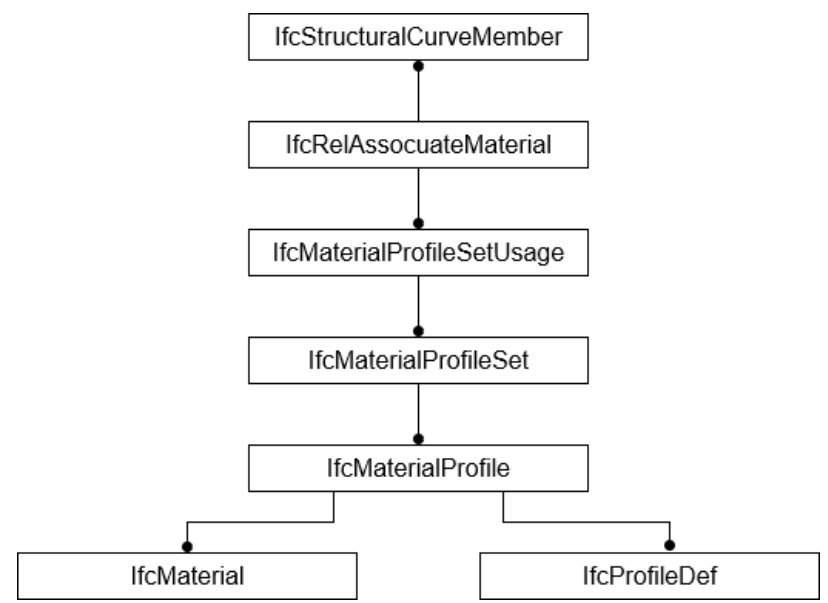

Figure 4: Referenced entities for material and profile definition in IfcStructuralCurveMember

\subsubsection{Wall, Plate and Slab}

Under the definition of IfcStructuralAnalysisModel, the plane building elements such as walls, plates and slabs are represented by IfcStructuralSurfaceMember that is a proper sub-type of IfcStructuralMember (buildingSMART 2020b). Unlike IfcStructuralCurveMember, in terms of structural analysis, data structure of the semantic model of IfcStructuralSurfaceMember consists of geometric definition (Figure 5) and material definition (Figure 6).

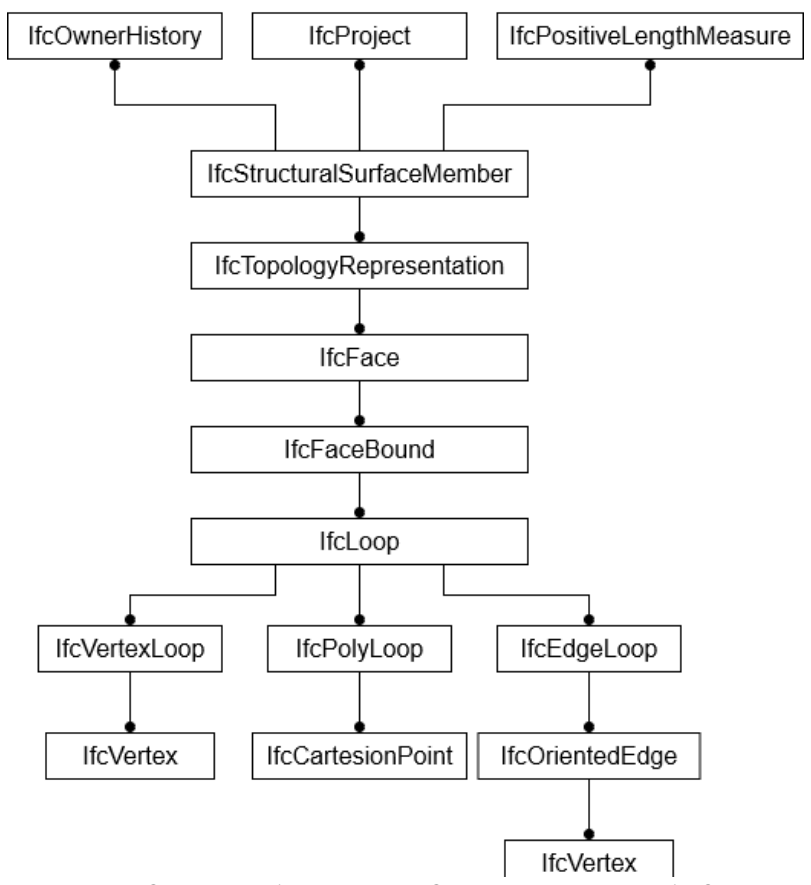

Figure 5: Referenced entities for geometric definition in IfcStructuralSurfaceMember 


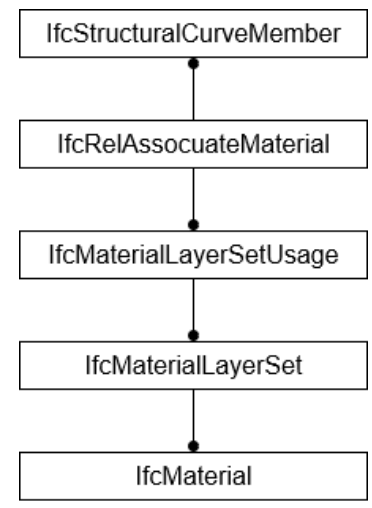

Figure 6: Referenced entities for material definition in IfcStructuralSurfaceMember

\subsection{Connection}

\subsubsection{Point Connection}

Point connection is realized through vertexes, it contains the coupling among curve members (beams and columns) or between curve members and surface members (walls, plates and slabs). The date structure of IfcStructralPointCnnection is illustrated in Figure 7. IfcVertextPoint storing global coordinates is referenced by IfcStructralPointoCnnection through IfcProductionDefinitionShape and IfcTopologyRepresentation. The point connection and the affiliated structural elements are separately objects defined in IFC, the entity IfcRelConnectsStructuralMember is applied to bridge the relation between these two objects. If a connection point defined by IfcStructralPointoConnection functions as a support, the connection then needs to be referenced to IfcBoudnaryNodeCondition, where 6 degrees of freedom in the 3D coordinate system are defined.

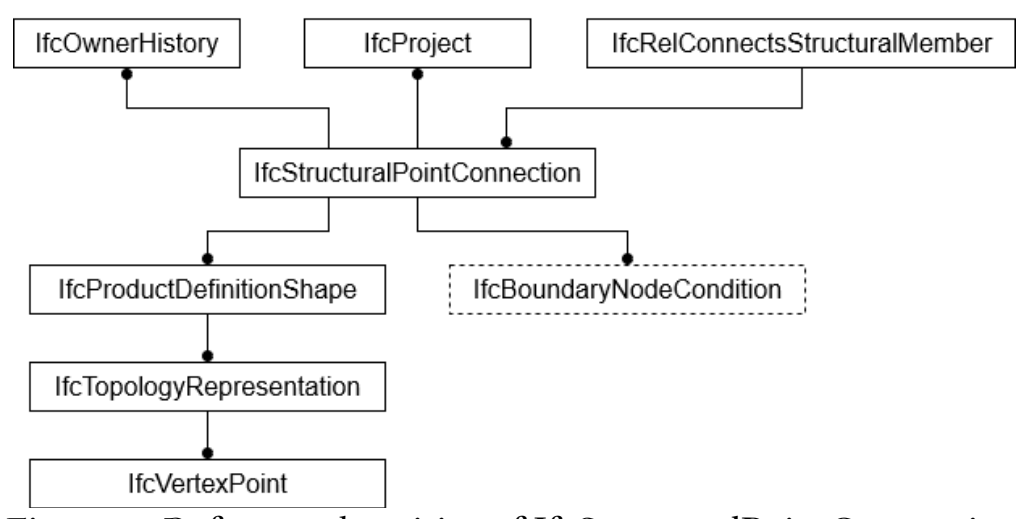

Figure 7: Referenced entities of IfcStructuralPointConnection

\subsubsection{Curve Connection}

The occurrence of curve connection exists in the shared edges of different surface members. Analogous to point connection, curve connection using IfcStructuralCurveConnection (Figure 8) comprises shape definition (IfcProductDefinitonShape) and boundary condition (IfcBoundaryEdgeCondition). The shared edge geometry is provided by IfcEdge composed with a starting and an end vertex. In a support point, 6 degrees of freedom can be either fixed or released in IfcBoundaryNodeCondition. The curve 
connection and its belonging structural members are required to be topologically referenced through IfcRelConnectsStructuralMemeber

as well.

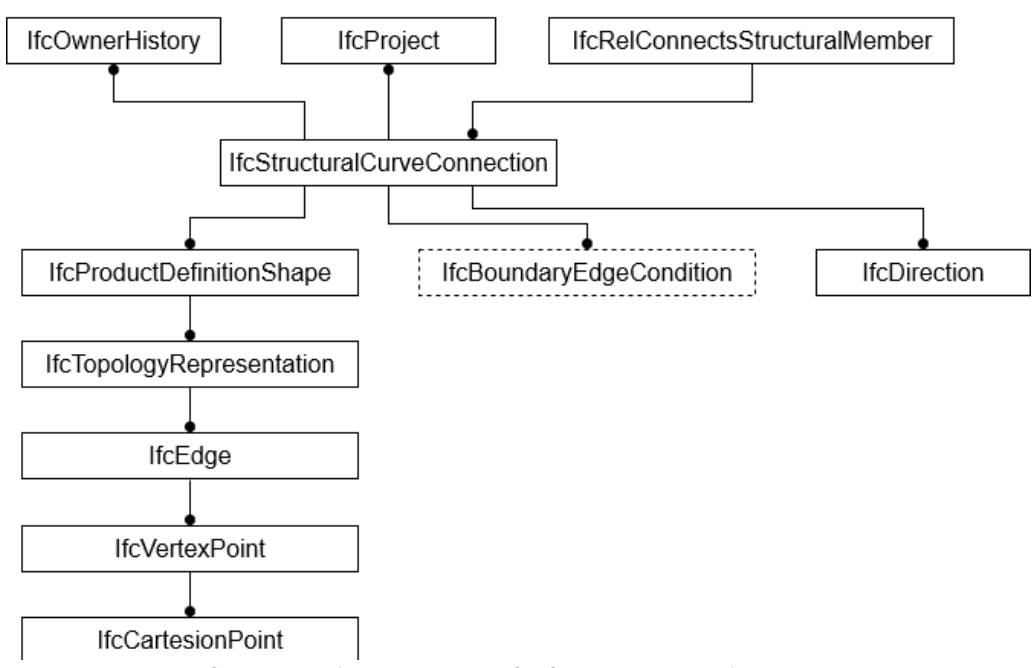

Figure 8: Referenced entities of IfcStructuralCurveConnection

\section{Finite-ELEMENT SYSTEM EsTABLISHMENT}

Generally, structural analysis contains static analysis and dynamic structural analysis. The governing numeric stiffness equation for static acting forces is

$$
\boldsymbol{K} \cdot \boldsymbol{u}=\boldsymbol{f}, \#(1)
$$

whereas the latter is numerically modelled through the motion (equilibrium) equation

$$
\boldsymbol{K} \cdot \boldsymbol{u}+\boldsymbol{D} \cdot \dot{\boldsymbol{u}}+\boldsymbol{M} \cdot \ddot{\boldsymbol{u}}=\boldsymbol{f} . \#(2)
$$

With respect to both equations, stiffness matrix $\mathrm{K}$ and mass matrix $M$ are sup-posed to be of major concern during data mapping. The damping matrix is in this research work omitted by reason of its complexity, which needs a separate paper to be developed properly. Considering that this paper concentrates on beam elements and surface elements, this section is dedicated to elaborating how a global stiffness matrix or a global mass matrix is established. Through investigating the derivation process of these two element matrices, the data requirements can be obtained for the purposed of semantic modelling towards structural analysis.

\subsection{Beam Element}

\subsubsection{Element Matrices}

Bernoulli-Euler Beam (Gavin 2018) is a classical and widely used hypothesis neglecting shear deformation and rotatory for beam element in structural analysis. A thin beam with a small cross section satisfies the principle of Bernoulli-Euler Beam. In a spatial coordinate system, each node of a beam element owns 6 degrees of freedom (Figure 9) including deformation along $\mathrm{x}$-axis (stretching), rotation around $\mathrm{x}$-axis (torsion), deformations along $\mathrm{y}^{-}$and $\mathrm{z}$-axis (deflection) and rotations around $\mathrm{y}^{-}$and $\mathrm{z}^{-}$axis (bending). Equation (3) and Equation (4) show the definition of an element stiffness matrix $\boldsymbol{K}_{e}$ and an element mass matrix $\boldsymbol{M}_{e}$ in a 3D coordinate system: 
and

$$
\boldsymbol{K}_{e}=E A \int_{0}^{l} \varphi^{\prime}{ }_{x} \varphi^{\prime}{ }_{x} d x+E I \int_{0}^{l} \varphi^{\prime \prime}{ }_{y}(x) \varphi^{\prime \prime}{ }_{y}(x) d x \#(3)
$$

$$
\boldsymbol{M}_{e}=\rho A \int_{0}^{l} \varphi_{x} \varphi_{x} d x+\rho A \int_{0}^{l} \varphi_{y}(x) \varphi_{y}(x) d x, \#(4)
$$

$E$ indicates Yong's modulus, $A$ area of element cross section, $I$ inertia moment and $l$ element length. Linear shape functions $\varphi_{x}$ is in the integration of the first term referring to stretching, Hermit-Polynomial serves as shape functions $\varphi_{y}$ in the integration of the second item about bending and torsion. One node has 6 degree of freedom, one beam element has 12 degree of freedom, thus an element matrix ought to have a size of $12 \times 12$.

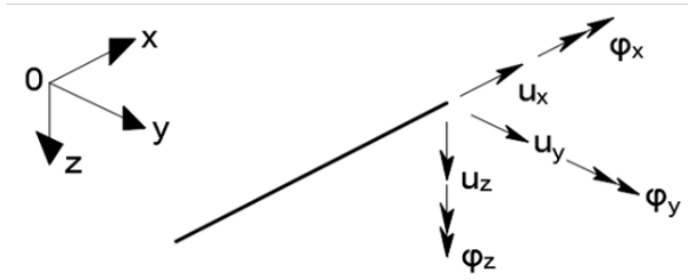

Figure 8: Six degrees of Freedom of a beam element in the 3D coordinate system

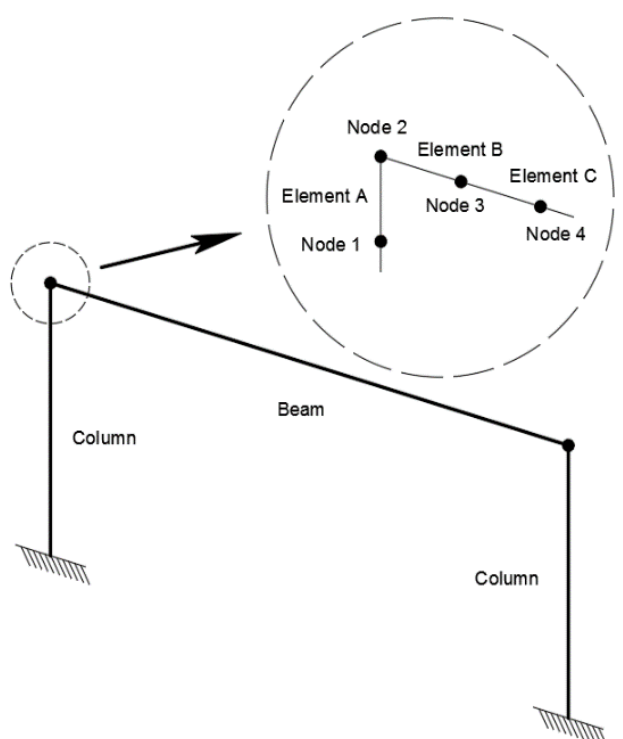

Figure 10: A frame with detail drawing at a connection part

\subsubsection{Global System}

As the detail drawing in Figure 10 illustrates, continuous modelling and coordinate transformation form a local and a global system are two issues in beam system construction. Adjacent beam elements are connected through shared nodes, this kind of connection is not only geometrical, but also material and mechanical. In the composite stiffness matrix of Element B and C (Figure 10) is represented in Equation (5); likewise, the corresponding mass matrix is established in the same way. The corresponding parameters of both elements are located at the position of Node 3.

$$
K / M=\left[\begin{array}{ccc}
\mid \text { Node 2(Elem. B) } \mid & 0 & 0 \\
0 & \mid \begin{array}{c}
\text { Node 3 (Elem. B })+ \\
\text { Node 3(Elem. C) }
\end{array} & 0 \\
0 & 0 & \mid \text { Node } 4 \text { (Elem.C) } \mid
\end{array}\right] \#(5)
$$


For a single column or a beam, all the interconnected beam elements are usually oriented to the same direction. However, in a more complicated structure like a frame composed by a beam and two column (Figure 10), Element A and Element B are perpendicular to each other, so that a simple overlapping of elements and nodes is not sufficient. As a consequence, the local coordinates of elements ought to be transformed to match the global coordinate system. The spatial transformation of a beam element is completed by rotations around the three axes in the global coordinate system. In Figure 11 , the beam element needed to be transformed is firstly rotated around z-axis from Equation (6), then y-axis from Equation (9), and eventually x-axis from Equation (10). The rotation angles $\varphi_{z}$ and $\varphi_{y}$ can be obtained by the nodal coordinates of an element, $\varphi_{x}$ is the rotation around x-axis, which is not in relation to the coordinates, but should be given externally, in the case of beam elements.

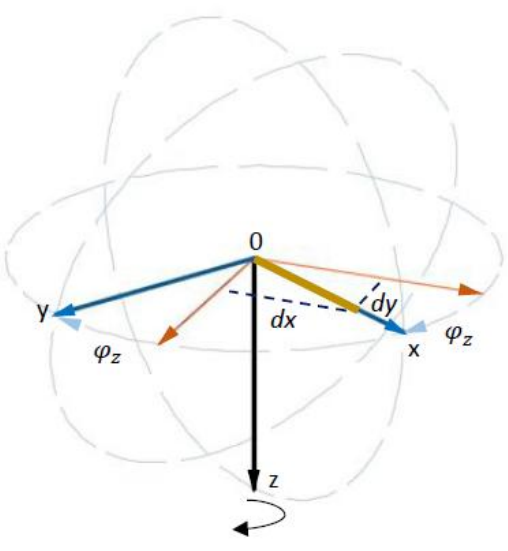

Step 1

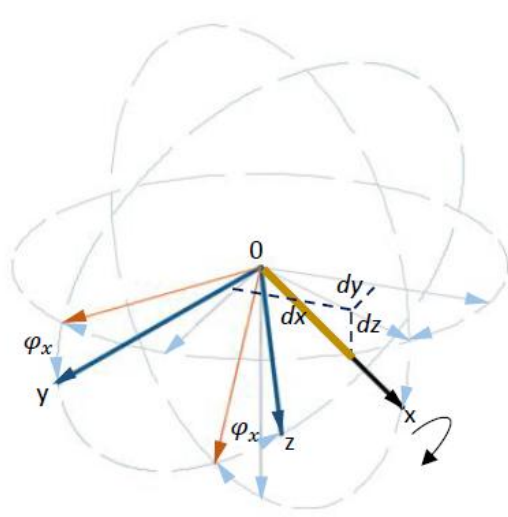

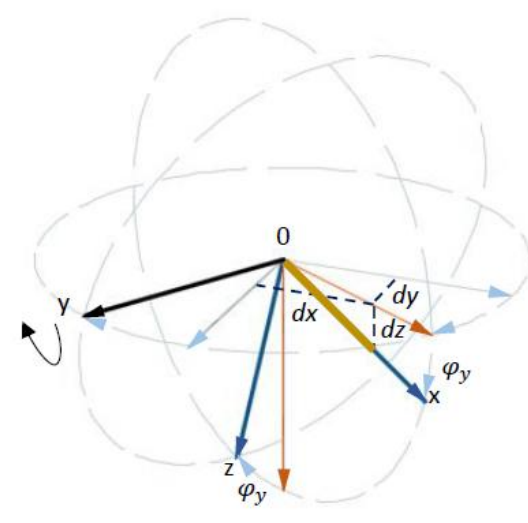

Step 2

Step 3

Figure 11: Coordinate transformation steps

$$
\begin{aligned}
t_{z}= & {\left[\begin{array}{ccc}
\cos \left(\varphi_{z}\right) & -\sin \left(\varphi_{z}\right) & 0 \\
-\sin \left(\varphi_{z}\right) & \cos \left(\varphi_{z}\right) & 0 \\
0 & 0 & 1
\end{array}\right] \#(6) } \\
\varphi_{z}=\arctan (d y / d x) \#(7) & \\
t_{y}= & {\left[\begin{array}{ccc}
\cos \left(\varphi_{y}\right) & 0 & \sin \left(\varphi_{y}\right) \\
0 & 1 & 0 \\
-\sin \left(\varphi_{y}\right) & 0 & \cos \left(\varphi_{y}\right)
\end{array}\right] \#(8) }
\end{aligned}
$$




$$
\begin{aligned}
\varphi_{y} & =\arctan \left(d z / \sqrt{(d x)^{2}+(d y)^{2}}\right) \#(9) \\
t_{x} & =\left[\begin{array}{ccc}
1 & 0 & 0 \\
0 & \cos \left(\varphi_{x}\right) & -\sin \left(\varphi_{x}\right) \\
0 & \sin \left(\varphi_{x}\right) & \cos \left(\varphi_{x}\right)
\end{array}\right] \#(10)
\end{aligned}
$$

The element transformation matrix $\boldsymbol{T}_{\boldsymbol{e}}$ from Equation 12 is, therefore, obtained from Equation (7), Equation (9) and Equation (11), and brought in to address this issue.

$$
\begin{gathered}
t=t_{z} \cdot t_{y} \cdot t_{x} \#(11) \\
\boldsymbol{T}_{e}=\left[\begin{array}{rrrr}
t & 0 & 0 & 0 \\
& t & 0 & 0 \\
s y m & t & 0 \\
s y m & & t
\end{array}\right] \#(12)
\end{gathered}
$$

\subsection{Surface Element}

As a matter of fact, the principle of surface finite element shows a more convoluted situation than beam element. Typical finite element types contain plane element, plate element and shell element, the complexity of the three element rises in turn. In addition, for a rectangular surface element, which is one of the most usually used surface element types for a regular-shape surface structural element is generally defined by 4 nodes. More nodes can be applied in surface element definition, which leads to a higher accuracy, but cause more complex element matrices and a larger and hence more expense in computing time. Triangular elements utilized widely for irregular-shape structural elements also share the identical features. Plane elements (Figure 12) can be employed to mesh a wall model, nevertheless is not suitable for slab and plate models that are usually meshed by finite plate or shell elements. This paper merely focuses on plane elements, other kinds of finite surface element will not be discussed.

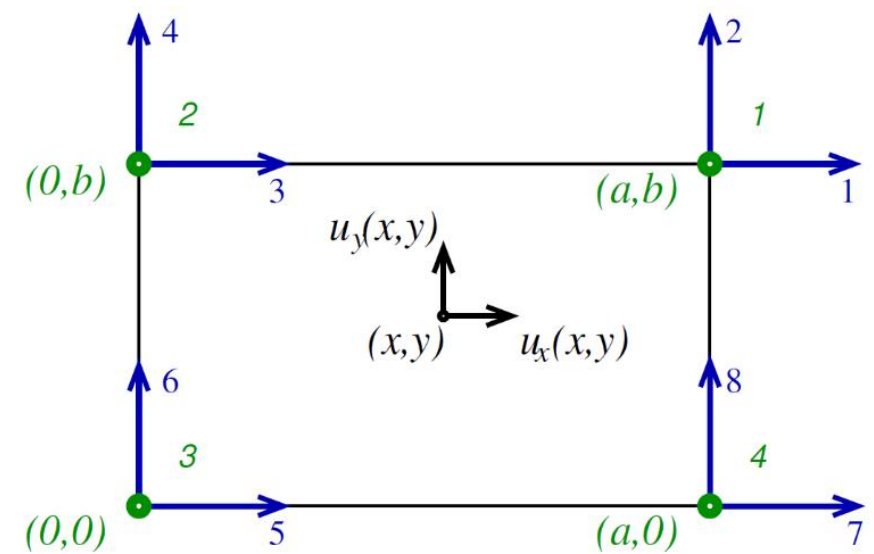

Figure 12: 2D rectangular element coordinates and displacements (Gavin 2018)

Under the basic assumption that the plane with uniform thickness are isotropic and homogeneous, a plane element is classified as plane-stress element or plane-strain element, according to their constitutive relationship. Both of the relation functions $\boldsymbol{S}_{p \sigma}$ and $\boldsymbol{S}_{p \epsilon}$, can be substituted in the numeric expression of element stiffness:

$$
\overline{\boldsymbol{K}}_{e}=\int_{A}\left[\boldsymbol{B}(x, y)^{T} \boldsymbol{S}(E, v) \boldsymbol{B}(x, y)\right] h d x d y, \#(13)
$$

And element mass:

$$
\overline{\boldsymbol{M}}_{e}=\int_{A} \rho\left[\boldsymbol{\Psi}(x, y)^{T} \boldsymbol{\Psi}(x, y)\right] h d x d y, \#(14)
$$


where $\overline{\boldsymbol{K}}_{e}$ indicates the plane element stiffness matrix, $\overline{\boldsymbol{M}}_{e}$ the plane element mass matrix, $h$ the uniform thickness, $\boldsymbol{S}$ the elastic relationship, $\boldsymbol{B}$ and $\boldsymbol{\Psi}$ are shape functions (e.g. linear functions, Hermite polynomials, etc.) for plane elements, in order to obtain the element matrices.

Two points are necessary to be emphasized. First off, plane elements are merely feasible for the surface elements under the loads parallel to the plane based on the original definition. In terms of surface building or structural elements, vertical walls satisfy the condition considering geometry and load directions, but sloping walls are not suitable for plane elements anymore. Secondly, the stiffness and mass matrices will have a size of $8 \times 8$, since then deformation of planes are limited on the $2 \mathrm{D}$ plane. In a 3D coordinates system, where each node has 6 degrees of freedom, the element matrices are extended to a size of $24 \times 24$ for the purpose of coupling between plane elements and beam elements.

\section{SEMANTIC MODELLING}

The instructions presented here have been used to structure this paper. In Figure 13, the semantic model towards structural analysis is illustrated by the Unified Modelling Language (UML), the UML diagram takes solely beam element and plane element into consideration, in addition it generically represents the topological relationship of them. According to the basic definition, one element has at least two nodes, conversely one node regarded as a coupling connection can be shared by several elements.

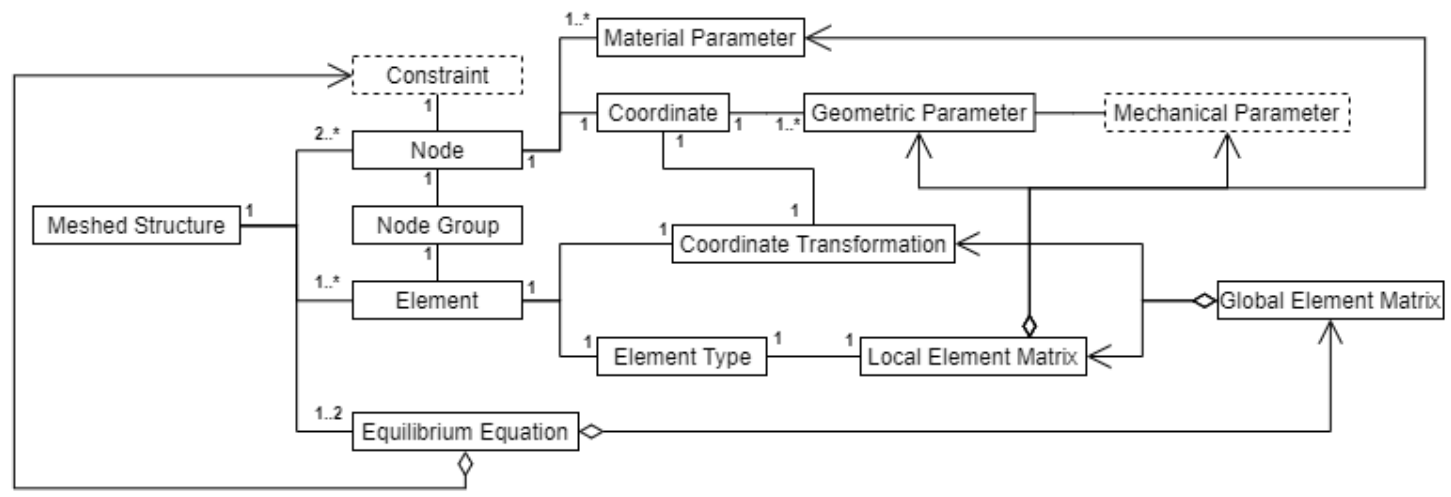

Figure 13: UML diagram of semantic model towards structural analysis using FEM

An element type (Figure 13) has two levels, the first level is dedicated to shape description of a finite element; the second implies the mathematical assumptions of finite elements, e.g. Bernoulli-Euler theory and Timoshenko theory for beam elements. Both layers lead directly to the definition of different terms in the local element stiffness and mass matrices. The nodal parameters includes material, geometrical and mechanical parameters, local coordinates and boundary conditions. They are all classified under the class Node in Figure 14. Geometrical parameters resulting from node coordinates contribute to calculate the material parameters. The boundary condition at the related node contains the states (fixed or released) of 6 degrees of freedom. Coordinate transformation is classified as an attribute of finite elements. Node coordinates are applied to obtain the spatial inclination angles of a finite element on three planes $(x-y$ plane, $y-z$ plane and $x-z$ plane) in a $3 D$ coordinate system. After deriving the local 
element matrices and transformation matrix, the global element matrices and successively the global matrices of the whole structure are able to be established.

\section{CONCEPTUAL CASE STUDY}

In order to verify the feasibility of proposed data mapping methodology, a beam model is discussed in this section as a conceptual case study. Figure 14 illustrates the required IFC entities and the semantically modelled objects for structural analysis to reflect the data flow during mapping.

IfcCartesianPoints serve as vertexes composing a beam structure. Coordinates in class Node (Figure 13) are utilized to locate elements and define their geometry. IfcDirection indicates the order of IfcCartensionPoints and successively nodes of finite elements. According to that, the class Node Group is assigned, so that classes Element and Node can be instantiated. Material parameters such as Yong's modulus stored in the entity IfcMaterial are mapped to the nodal Material Parameters. Mechanical Parameter of cross section of beam elements can be designated by the values from the entity IfcProfileDef, or derived directly based on the measure of cross section, which should be automatically attached through referenced entities of IfcProfileDef, when an IFC model is exported from a BIM software application. The attributes defined in IfcBoundaryConidtion referring to constraints in FEM can be converted to the numeric representationof degrees of freedom at nodes. Eventually, the data obtained from the IFC model is employed to establish the stiffness and mass matrices (Section 3) for the purpose of structural analysis.

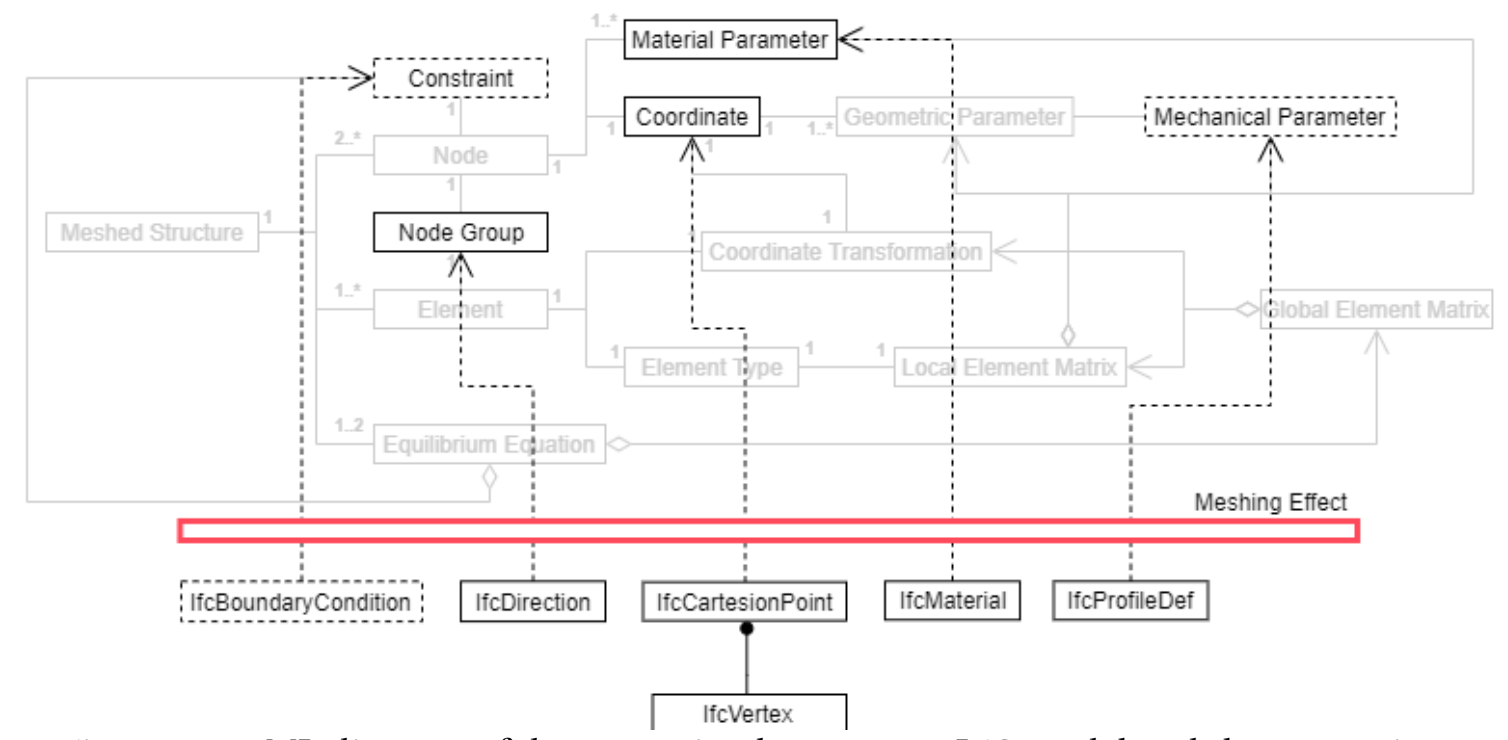

Figure 14: UML diagram of data mapping between an IFC model and the semantic model

\section{CONCLUSIONS}

This paper presents an overview of data application from IFC models with various application domains and addresses a specific concern in the field of structural analysis. A methodology is conceived to map data from IFC models to FE systems for structural analysis. Data architecture of IFC standard is analysed to comprehend modelling 
philosophy in the domain of structural analysis. Concurrently, data requirements of FE equilibrium equations are also studied in order to construct a topological representation semantically between structures, finite elements, nodes and the related parameters. The semantic model shows a relatively generic schema for beam elements and surface element. Focusing on beam structures, the proposed data mapping methodology is implemented with a conceptual case study, where the meshing effect is beard in mind, to bridge the gap between an IFC mode and the finite element system. Since this paper presents a theoretical research work, the methodology is expected to be enriched towards data mapping for other kinds of beam elements and surface element in future work.

\section{ACKNOWLEDGMENTS}

This research work belongs to an extended study from the project wiSIB, No. 01IS16031C. The authors would like to acknowledge the support of the Federal Ministry of Education and Research of Germany to the funding of this project.

\section{REFERENCES}

Ahn, K.-U., Kim, Y.-J., Park, C.-S., Kim, I. and Lee, K. (2014). BIM interface for full vs. semi-automated building energy simulation. Energy and Buildings, 68, pp.671-678.

Andriamamonjy, A., Saelens, D. and Klein, R. (2018). An automated IFC-based workflow for building energy performance simulation with Modelica. Automation in Construction, 91, pp.166-181.

buildingSMART. (2020a). buildingSMART - The Home of BIM [online]. Available from: https://www.buildingsmart.org/ [accessed 23 April 2020].

buildingSMART. (2020b). IFC4 Documentation. Available from: https://standards.buildingsmart.org/IFC/DEV/IFC4_2/FINAL/HTML/.

Choi, J.W., Kim, S.A., Lertlakkhanakul, J. and Yeom, J.H. (2008). Developing Ubiquitous Space Information Model for Indoor GIS Service in Ubicomp Environment. In: 2008 Fourth International Conference on Networked Computing and Advanced Information Management. 2008 Fourth International Conference on Networked Computing and Advanced Information Management. Gyeongju, Korea: IEEE Computer Society 2008, pp.381-388.

Dimyadi, J., Spearpoint, M. and Amor, R. (2008). Sharing Building Information using the IFC Data Model for FDS Fire Simulation. Fire Safety Science, 9, pp.1329-1340.

Fu, C., Aouad, G., Lee, A., Mashall-Ponting, A. and Wu, S. (2006). IFC model viewer to support $\mathrm{nD}$ model application. Automation in Construction, 15(2), pp.178-185.

Gavin, H.P. (2018). CEE 541. Structural Dynamics. , 2018. Available from: http://people.duke.edu/ hpgavin/cee541/ [accessed 30 January 2020].

GRAPHISOFT. (2020). What is OPEN BIM and why should you care? - Information about OPEN Building Information Modeling and its importance [online]. Available from: https://www.graphisoft.com/archicad/open_bim/ [accessed 30 January 2020].

Hamdan, A.-H. (2018). Generierung eines Tragwerkmodells basierend auf IFC-Daten für geschädigte Brücken. In: Proceeding of the 30th Forum Bauinformatik. Forum Bauinformatik 2018. Weimar, Germany: Bauhaus-Universität Weimar.

Hu, Z.-Z., Zhang, X.-Y., Wang, H.-W. and Kassem, M. (2016). Improving interoperability between architectural and structural design models: An industry foundation classesbased approach with web-based tools. Automation in Construction, 66, pp.29-42. 
Motamedi, A., Soltani, M.M., Setayeshgar, S. and Hammad, A. (2016). Extending IFC to incorporate information of RFID tags attached to building elements. Advanced Engineering Informatics, 30(1), pp.39-53.

Quirk, V. (2012). A Brief History of BIM [online]. ArchDaily [online]. Available from: http://www.archdaily.com/302490/a-brief-history-of-bim/ [accessed 5 January 2020].

Romberg, R., Niggl, A., Treeck, C. van and Rank, E. (2004). Structural Analysis based on the Product Model Standard IFC.

Stascheit, J., Meschke, G., Koch, C., Hegeman, F. and König, M. (2013). Process Oriented Numerical Simulation of Mechanized Tunnelling Using an IFC-Based Tunnel Product Model. In: Proceedings of the 13th International Conference on Construction Applications of Virtual Reality (CONVR). International Conference on Construction Applications of Virtual Reality. London, UK, p.8.

Thiis, T.K. and Hjelseth, E. (2008). Use of BIM and GIS to enable climatic adaptations of buildings. In: 7th European Conference on Product and Process Modelling (ECPPM). European Conference on Product \& Process Modelling. Sophia Antipolis, France.

Weise M., Katranuschkov P., Liebich T., Scherer R.J., Structural Analysis Extension of the IFC Modelling Framework, e-journal ITcon, Volume 8, pg. 181 - 200, 2003.

Yamamura, S., Fan, L. and Suzuki, Y. (2017). Assessment of Urban Energy Performance through Integration of BIM and GIS for Smart City Planning. Procedia Engineering, 180, pp.1462-1472. 